\title{
Supramolecular Structures and Self-Association Processes in Polymer Systems
}

\author{
M. HRUBÝ ${ }^{1}$, S. K. FILIPPOV ${ }^{1}$, P. ŠTĚPÁNEK ${ }^{1}$ \\ ${ }^{1}$ Institute of Macromolecular Chemistry of the Czech Academy of Sciences, Prague, Czech \\ Republic
}

Received July 14, 2016

Accepted July 14, 2016

\section{Summary}

Self-organization in a polymer system appears when a balance is achieved between long-range repulsive and short-range attractive forces between the chemically different building blocks. Block copolymers forming supramolecular assemblies in aqueous media represent materials which are extremely useful for the construction of drug delivery systems especially for cancer applications. Such formulations suppress unwanted physicochemical properties of the encapsulated drugs, modify biodistribution of the drugs towards targeted delivery into tissue of interest and allow triggered release of the active cargo. In this review, we focus on general principles of polymer selforganization in solution, phase separation in polymer systems (driven by external stimuli, especially by changes in temperature, $\mathrm{pH}$, solvent change and light) and on effects of copolymer architecture on the self-assembly process.

\section{Key words}

Copolymers • Nanoparticles • Phase separation • Responsivity

\section{Corresponding author}

P. Štěpánek, Institute of Macromolecular Chemistry of the Czech Academy of Sciences, Heyrovského nám. 2, 16206 Prague 6, Czech Republic. Fax: +420 296809 410. E-mail: stepan@imc.cas.cz

\section{Introduction}

Self-organisation in a polymer system appears when a balance is achieved between long-range repulsive and short-range attractive forces between the chemically different building blocks. The increasing capacity to synthesize macromolecules (Hadjichristidis et al. 2002) with more complex and controlled composition, along with a better understanding of the physical-chemical processes interactions have provided the basis not only for a rational design and fabrication of sophisticated selfassembled nanostructures but, more generally, the basis for a new and powerful nanotechnology (Forster and Konrad 2003). In particular, the development of selfassembling block copolymers with different blocks being able to segregate in immiscible phases under appropriate conditions, have proven their enormous potential to obtain different nanostructures of interest, both in 2 and 3 dimensions (Hamley 1999). This naturally leads to investigation of physical (Strobl 2007) and biophysical aspects of self-assembled polymer materials since selforganization is the governing principle of many biophysical activities and structures.

Both biotechnology and pharmaceutical industries have produced wide variety of new medical treatments based on the key biomacromolecules: DNA, RNA, peptides and proteins. While these therapeutics have tremendous potential, developing effective delivery formulations is a great challenge. Among the important factors in the successful clinical application of such drugs is securing of their sufficient stability, administration, absorption, metabolism and bioavailability at the target site. Polymer or biopolymer nanoparticles, which are formed by association of various macromolecules, are frequently used for drug delivery purposes (Kabanov et al. 1998). The formation of suitable nanoparticles should be properly sensitive to the actual bio-environment which is usually achieved by adequate changes in the 
conformation, shape, hydrophilicity of building polymers, etc. Therefore, stimuli-responsive, "intelligent" or "smart" polymers that respond with large property changes to small physical or chemical stimuli have been frequently investigated (Hoffman 1997, Park 1997, Swarbich 1994, Jeong et al. 2002, Leroux et al. 2001, Lazzari et al. 2006). The main impetus for studying these polymers has been the need for a better understanding of the physical mechanism of co-operative interactions in biopolymers which, in turn, stimulated the research of mimicking the co-operative behavior in synthetic systems. Stimuli-responsive polymers were developed, which respond in a desired way to a change in temperature, $\mathrm{pH}$, electric or magnetic field, or in another parameter. "Smart" polymers can be used as an active part of stimuli-responsive nanoparticles formed by selfassociation of macromolecules.

Self-assembled nanoparticles can be categorized in different ways; a useful classification is according to the dominant type of polymer-polymer interaction where five major categories of nanoparticles can be distinguished:

(1) stereocomplexes formed via van der Waals forces;

(2) polyelectrolyte (or polyionic) complexes which are formed between polymer acids and bases or their salts and are stabilized by ionic bonds;

(3) complexes formed by hydrogen bonding between polyelectrolytes and non-ionic polymers;

(4) micelles, vesicles and other micelle-like nanoparticles formed by hydrophobic interactions.

Some nanoparticles exhibit a combination of these phenomena. Interpolymer nanoparticles often display physical and biological properties very different from those of individual constituent polymers.

Very commonly used structure-forming polymeric materials are block copolymers, in particular diblock copolymers where the long-range repulsion interaction is given by the incompatibility of the blocks and the short-range attraction is provided by the covalent bond between the blocks (Forster and Konrad 2003, Hadjichristidis et al. 2002). In the presence of solvent in the system several types of self-assembled structures exist the structure of which is governed by the thermodynamic interactions between the solvent and the blocks of the copolymer. A classic example is a micellar structure of a diblock copolymer solution occurring when the solvent is selective (thermodynamically good for one block and bad for the second block); other types of possible common arrangements are polymersomes or layered structures.

The common approach applied to describing such structures is based on controlling the extent of macrophase separation that occurs in a mixture of two compounds (solvents or polymers) that became immiscible or incompatible as a result of a change of an external variable. This parameter can be temperature, $\mathrm{pH}$ or addition of a co-solvent, in principle it could also be a change in pressure but the latter is not very practical since usually large pressure changes are needed to achieve relatively small changes in phase diagrams due to low compressibility of common liquids.

A phase diagram for a polymer $\mathrm{A} /$ polymer $\mathrm{B}$ or polymer/solvent system is schematically represented in Figure 1. In the classical case the energy of the system is given by enthalpic and entropic contributions and the interaction parameter $\chi$ is given by the Flory-Huggins relation:

$$
\chi=a / T+b
$$

where $\mathrm{T}$ is absolute temperature and $a$ and $b$ are specific for the polymer/polymer or polymer/solvent pair. For more complex systems, a third term $\chi_{\mathrm{s}}$ has to be included:

$$
\chi=a / T+b+\chi_{s}
$$

In this simple representation the third term includes all additional interactions in the system, in particular the effect of different temperature expansions of the system components and that of various specific interactions in the system (hydrogen bonds, ionic interactions, ...) that may be dominant compared to the enthalpic/entropic terms $a, b$.

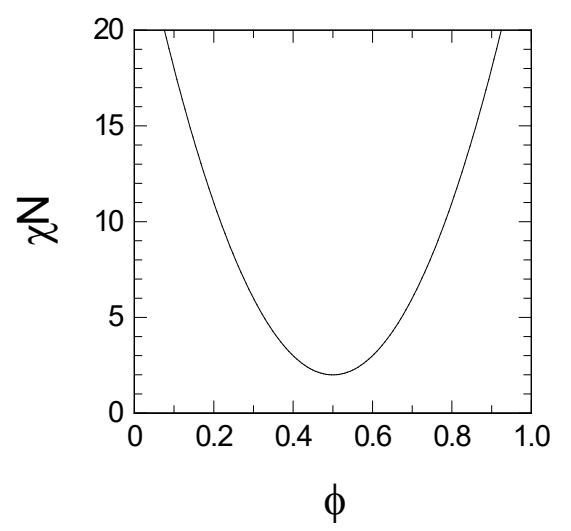

Fig. 1. A schematic phase diagram for a polymer $A /$ polymer $B$ or polymer/solvent. $\phi$ is the volume fraction of the first component of the system, $\mathrm{N}$ the number of monomeric units in the polymer chain and $\chi$ the interaction parameter describing the strength of interaction between polymer $\mathrm{A}$ and $\mathrm{B}$ or between the polymer and solvent. 
Once a macrophase separation has been initiated, the spatial extent of inhomogeneities produced by nucleation or spinodal decomposition can be controlled and/or limited by accumulated charge on the surface of growing nuclei preventing macrophase separation due to electrostatic repulsion or by addition of amphiphilic molecules chosen in such a way that one part of this amphiphile interacts attractively with the nucleated material while the other part interacts attractively with the surrounding solvent. If the amount of added amphiphiles is sufficient, the surface of the nucleated material then becomes covered with the amphiphilic molecules which effectively terminates the phase separation and stabilizes the system in a dispersed state. The formation of nanoparticles is always driven by thermodynamics (increase of $\chi$-parameter in Eq. (1)) but controlled by specific factors of interaction with the amphiphilic molecules including the composition ratio of a polymer and amphiphile, kinetic and hydrodynamic effects. Depending on composition of the system this dispersed state can be in the form of particles or three-dimensional structures.

\section{Phase separation}

Next we discuss the main driving forces in a polymer solution that lead to phase separation and formation of nanoparticles.

Phase separation induced by a change in temperature: Stimuli-responsive polymeric nanoparticles formed by hydrophobic interaction and stabilized by surfactants

In aqueous environment, some of the polymers and their hydrogels undergo fast, reversible changes in microstructure from a hydrophilic to a hydrophobic state. The changes are triggered by small changes of the environment but are apparent at the macroscopic level as precipitation or order-of-magnitude changes in the size and water content of hydrogels. Highly non-linear responsive polymer systems occurring in water are ideal candidates for the use in biotechnology or medicine. Stimuli-responsive polymers are already used in separation membranes (Jeong et al. 2002, Taylor and Cerenkowski 1975), drug delivery systems (Kohori et al. 1998, Leroux et al. 2001), cell cultures (Okano et al. 1995), for isolation of biomolecules (Monji and Hoffman 1987, Liang et al. 1999) and enzyme activity control (Park and Hoffman 1988). Thus the "smart" polymers can be used as an active part of stimuli-responsive nanoparticles formed by association of the macromolecules.

The most often studied polymers are thermosensitive polymers where the phase separation is achieved by a change in temperature $\mathrm{T}$ in Eq. (1): poly(Nisopropyl acrylamide) (PNIPAM) (Aseyev et al. 2006, Kujawa et al. 2006, Tailor and Cerenkowski 1975) poly(N-vinylcaprolactam) and poly(methyl vinyl ether) (Aseyev et al. 2006, Kujawa et al. 2006) and their copolymers with either hydrophobic or hydrophilic monomers and polymers. PNIPAM is a water-soluble polymer with many attractive properties. It undergoes a coil-to-globule transition upon heating at $32{ }^{\circ} \mathrm{C}$ (lower critical solution temperature, LCST (Saiyto et al. 1993)), a property that is the result of rather complex polarity of this molecule. Below LCST, the amide groups bind water molecules via hydrogen bonding, thus imparting to the polymer both water solubility and surface activity. Above the transition temperature, the hydrogen bonds break, the polymer expels water molecules and undergoes a coil-toglobule transition. The LCST close to physiological temperature makes the polymer an especially good candidate for applications in biotechnology and in medicine (Aseyev et al. 2006, Wang and Wu 1999, Oupický et al. 2003).

As the phase separation of PNIPAM-based systems has been frequently studied in cross-linked aqueous gels (volume-phase separation (Hirotsu et al. 1987)) and solutions of linear polymers (Schild 1992, Chytrý et al. 1997, Cammas et al. 1997), attention has been also paid to the thermally sensitive association of copolymers containing blocks or grafts of PNIPAM. Recently, we have prepared well defined nanoparticles of PNIPAM on heating above the LCST in presence of ionic and non-ionic surfactants (Konak and Hruby 2006, Konak et al. 2007) in particular SDS (sodium dodecyl sulphate). The effect of PNIPAM and surfactant concentration, and molecular weight of PNIPAM on nanoparticle parameters and on the phase transition temperature of PNIPAM solutions was investigated.

The observed formation of well-defined thermosensitive nanoparticles can be qualitatively explained by the following model (Konak and Hruby 2006). The nuclei of a condensed phase formed after the polymer solution passes through the phase transition temperature are monodisperse at the early stage of phase separation as established in the theory of nucleation and growth (Cahn 1956, Christian 1981). They are hydrophobic and, therefore, they attract surfactants 
molecules. When the adsorbed surfactant reaches a critical surface concentration, the nanoparticles are sufficiently solubilized and the growth of nanoparticles stops. The proposed mechanism of nanoparticle formation is shown in Figure 2.

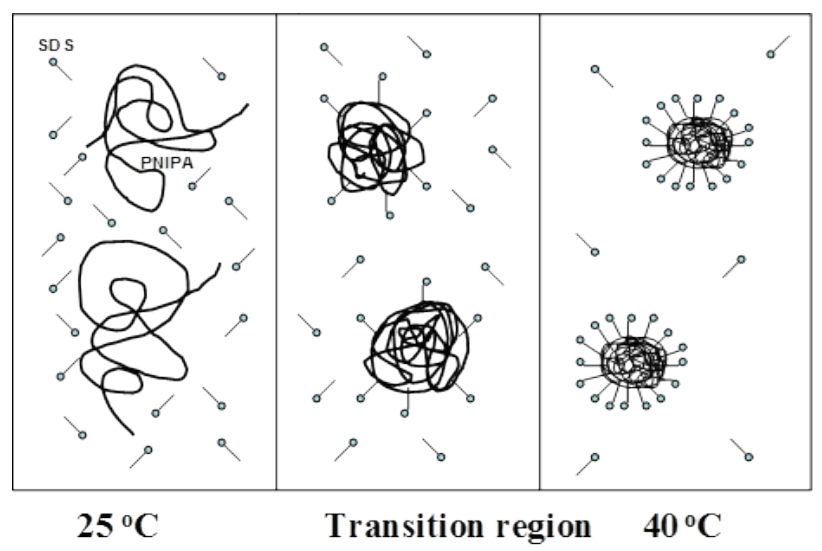

Fig. 2. Schematic representation of nanoparticle formation by precipitation of PNIPAM when heated above the LCST and by interaction with surfactant molecules of SDS.

\section{Phase separation induced by a change in $p H$}

Several groups reported investigations of micellar nanoparticles composed of $\mathrm{pH}$-responsive polymers (Lee et al. 2005, Licciardi et al. 2006, Sakai et al. 2006). A biologically active unit (drug) is released by disassembling the micelle in response to the change of the external $\mathrm{pH}$. The $\mathrm{pH}$-dependent disassembly of the micelles is in the most cases given by the presence of an ionizable group (a weak acid or a weak base) on the relatively hydrophobic polymer forming the micelle core. When the external $\mathrm{pH}$ increases, ionization degree of the polymer may increase (weak polyacid) or decrease (weak polybase). In the case of relatively hydrophobic polymer backbone, changing ionization degree strongly influences the hydrophilic-hydrophobic balance since ionized forms are always much more hydrophilic than the corresponding nonionized species because electrostatic interactions are stronger than hydrophobic ones. Such an increase of polarity due to the increase in ionization degree finally leads to the suppression of the hydrophobic forces which drive the polymer assembly and a reversible pH-dependent disassembly occurs. In addition to it, the polymer chains becoming ionized to the same charge coulombically repel each other further contributing to disassembly of the micelle. A weak hydrophobic polyacid as a micelle core-forming block thus disassembles when $\mathrm{pH}$ is increased and vice versa.
Exploiting this behavior we have recently tested the interaction with amphiphilic molecules to construct polymeric nanoparticles. The $\mathrm{pH}$ of the solution is gradually changed so that the polymer which is in the beginning in an environment where it is molecularly soluble (i.e. in the mostly ionized form), starts to precipitate. Adding a surfactant terminates the phase separation in a controlled way leading to formation of well-defined nanoparticles with low polydispersity. We have demonstrated this procedure (Filippov et al. 2008) using a $\mathrm{pH}$-sensitive hydrophobic polymer - poly $(\mathrm{N}-$ methacryloyl-L-valine) (PNMV), the extent of macrophase separation was controlled by the amphiphilic molecule Brij98 (Polyoxyethylene (20) oleyl ether). We have shown that in a certain range of concentration and composition of the polymer/amphiphile system very monodisperse particles with size ca. $50 \mathrm{~nm}$ could be reproducibly prepared after a change of $\mathrm{pH}$ from 7 to 3.5 . This change is reversible and the nanoparticles can be repeatedly created and dissolved by changes in $\mathrm{pH}$. This type of particles can be very useful, since they may be able to solubilize hydrophobic drugs in large amounts and release them after a change of $\mathrm{pH}$. For example the $\mathrm{pH}$ of stomach is 1 to 3 (nanoparticles associated), while the $\mathrm{pH}$ of duodenum is 7 to 8 (particles dissolved, drug released).

More detailed study of poly(N-methacryloyl-1valine), poly(N-methacryloyl-1-phenylalanine), and poly(N-methacryloylglycine-1-leucine) with Brij98 conducted by isothermal titration calorimetry, electrophoretic measurements, small-angle neutron scattering, and infrared spectroscopy methods revealed that nanoparticles could have either "pearl-necklace" or "core-shell" structure depending on the amino acid structure in the side chain of the polymer (Bogomolova et al. 2014).

\section{Phase separation induced by a change of solvent}

Polymeric nanoparticles stabilized by surfactants can be also created by acting on the parameters $a$ and $b$ in Eq. (1). These are system specific so that, e.g. for a polymer/solvent system phase separation is induced by a change of the solvent. We have successfully tested this procedure: polymeric nanoparticles were prepared by mixing a polymer - poly(methyl methacrylate) or polystyrene - dissolved in a water-miscible organic solvent with an aqueous solution of a surfactant (sodium dodecyl sulfate, SDS). Since water is a thermodynamically bad solvent for either of the polymers they start to precipitate but the presence of a surfactant terminates the phase 
separation and nearly monodisperse nanoparticles appear with a typical size in the range of 50 to $100 \mathrm{~nm}$. Finally the organic solvent more volatile than water is evaporated.

\section{Photoinduced phase separation.}

A different way of acting on the parameters $\mathrm{a}$ and $\mathrm{b}$ in Eq. (1) is a change in the polymer itself. Photoinduced changes of polymer configuration belong to this category. A good example of this situation is azobenzene that in normal condition is in a E configuration, after illumination with ultraviolet light (UV) switches to $\mathrm{Z}$ configuration. Azobenzene can be introduced in a polymer as a side chain, e.g. into poly[N(2-hydroxypropyl)methacrylamide] (Konak et al. 1994) or poly[2-(dimethylamino)ethyl methacrylate] (Lee et al. 2006). The $\mathrm{E}$ isomer is planar and practically apolar, while the $\mathrm{Z}$ isomer is not planar, bears a high dipole moment and shows higher solubility in polar solvents, e.g. in water. With an appropriate content of azobenzene this polymer can become more soluble in water after irradiation with UV light. Polymers can be prepared where illumination both increases or decreases solubility in water (Shimoboji et al. 2002) - copolymers of dimethyl acrylamide and phenylazophenyl acrylamide exhibit a decrease of LCST in water while copolymers of dimethyl acrylamide and phenylazophenyl acrylate exhibit an increase of LCST. Analogous effects of illumination on polymer solubility have been observed for spiropyrane containing polymers, where under UV light noncharged spiropyrane is transformed into zwitterionic merocyanine insoluble in polar solvents (Konak et al. 1997). Light-sensitive micellization has been also reported for diblock copolymers of azobenzenecontaining polymethacrylate and poly(tert-butyl acrylate) (Wang et al. 2004). Photosensitive Z-E isomerisation was also reported in stilbene-modified poly(methyl methacrylate) polymers ( $\mathrm{Yu}$ et al. 2006). Photoinduced phase separation or dissolution is however limited to polymers that contain a photosensitive group.

\section{Block copolymer nanoparticles}

The size of block copolymer micelles is dependent on the polymer characteristics and ranges usually (Kataoka et al. 2001) from 10 to $100 \mathrm{~nm}$. Therefore, lipophilic agents, such as hydrophobic drugs, can by solubilized in the micellar core, significantly affecting its concentration in aqueous media. The encapsulation efficiency is dependent on several parameters, including drug and polymer solubility parameters, size, shape and physical state of the hydrophobic guest molecule, as well as block copolymer length and volume ratio.

Many micelles with a thermoresponsive core and hydrophilic corona have the additional advantage in that they can be simply prepared by heating the thermoresponsive polymer in aqueous solution to a temperature above their cloud-point temperature, CPT (Hruby et al. 2009, Hruby, Filippov et al. 2010a, Hruby, Filippov et al. 2010b). Among polymeric micellar drug delivery systems, poly(ethylene oxide), PEO, is most often employed because of its high hydrophilicity and biocompatibility, long experimental record, and FDA approval (Torchilin 2002). However, careful studies of PEO-derived systems over recent decades have revealed some drawbacks, namely, interactions with various immunological entities and superfluous accumulation in the body (Knop et al. 2010). Another important reason for the search for alternatives is the wide range of patent coverage for nearly all PEO drug carriers and therapeutics. The PEO alternatives poly[N-(2hydroxypropyl)methacrylamide], HPMA, polyvinylpyrrolidone, PVP, and poly(2-oxazoline), POX, have been examined (Sedlacek et al. 2012).

Because the micelle is supramolecular aggregate of tens to hundreds of macromolecules, one can easily construct hybrid micelles containing several types of moieties and polymer blocks in desired ratio by simple mixing several appropriate block copolymers and forming mixed micelle afterwards from such mixture. The only prerequisite for formation of such macromolecular modular toolbox is compatibility of the copolymers with each other, which may be assured by, e.g. identical hydrophobic core-forming block.

Macromolecular drug delivery systems attract tremendously ascending interest in contemporary drug research, because they offer many possibilities of suppressing unfavourable properties of low-molecularweight drugs while enhancing the requested ones (Jain 2000). Polymeric micellar drug delivery systems (Kwon and Kataoka 1995, Kwon and Okano 1996) of core-shell architecture based on amphiphilic AB diblocks, ABA triblocks or graft copolymers have numerous advantages. They are easy to prepare and could be used for all hydrophobic drugs even if they do not contain suitable functional groups for covalent attachment, do not require chemical modification of the drug (advantageous due to 
regulatory issues), but also possess some disadvantages. This is especially the case of the drug release rate that is often hard to control and modify.

Systems with a covalently bound drug should be able to overcome these difficulties if a stimuli-responsive cleavable bond between polymer and drug is used, although such systems are harder to prepare and thus they are much less studied. In particular $\mathrm{pH}$ sensitive bonds cleavable under mildly acidic conditions and stable under neutral conditions (e.g. pH labile hydrazone bond) are studied in such cases, (Yoo and Park 2001, Kataoka et al. 2000, Yoo et al. 2002) because the $\mathrm{pH}$ value of the interstitial space of solid tumors as well as the interior of endosomes is usually more acidic ( $\mathrm{pH}$ close to 5) than blood plasma ( $\mathrm{pH}$ 7.4) (Kataoka et al. 2000). Very promising are also reductively biodegradable micelles of copolymers containing disulfide bonds. These micelles undergo degradation by reduced glutathione followed by drug release inside living cells where reduced glutathione is present in concentrations $0.1-10 \mathrm{mmol} / \mathrm{l}$. In contrary to that, outside the cells, glutathione is mostly in its oxidized form and in micromolar concentrations only (Kwon and Kataoka 1995).

Recently, we have successfully investigated two such biodegradable micellar systems; $\mathrm{pH}$ sensitive micelles (Hruby et al. 2005a, b) and nanoparticles biodegradable by the action of glutathione (Hruby et al. 2007). Thermoresponsive polymeric micelles and nanoparticles with thermoresponsive core and hydrophilic corona offer even several additional advantages over simple non-stimuli-responsive micelles for anticancer therapy. Firstly, they are much easier to prepare, e.g. by simple heating of aqueous solution of the block or graft copolymer from room to body temperature. Secondly, controlled hydrophilization of the core during hydrolytical degradation of the thermoresponsive block may provide extra control over micelle degradation and may serve also for drug release, if the hydrophobic part is a drug in the same time; this is the case of, e.g. doxorubicin.

A novel polymeric micellar pH-sensitive system for delivery of doxorubicin (DOX) was prepared (Hruby et al. 2005a, Hruby et al. 2005b) by self-assembly of amphiphilic diblock copolymers in aqueous solutions. The copolymers consist of a biocompatible hydrophilic poly(ethylene oxide) (PEO) block and a hydrophobic block containing covalently bound anthracycline antibiotic DOX. The starting block copolymers poly(ethylene oxide)-block-poly(allyl glycidyl ether)
(PEO-PAGE) with a very narrow molar-mass dispersity $\left(M_{w} / M_{n}\right.$ ca. 1.05) were prepared by anionic ring opening polymerization using sodium salt of poly(ethylene oxide) monomethyl ether as macroinitiator and allyl glycidyl ether as functional monomer that were further functionalized to form polymer hydrazide. The hydrazide was coupled with DOX yielding $\mathrm{pH}$-sensitive hydrazone bonds between the drug and carrier. The resulting conjugate forms micelles with $R_{h} \sim 100 \mathrm{~nm}$ in phosphatebuffered saline.

Thermoresponsive polymer micelles for applications as drug and radionuclide carriers were prepared from ABA triblock copolymers poly[2-methyl2-oxazoline-block-(2-isopropyl-2-oxazoline-co-2-butyl2-oxazoline)-block-2-methyl-2-oxazoline]. These polymers are molecularly dissolved in aqueous milieu below the cloud point temperature (CPT) of the thermoresponsive central poly(2-isopropyl-2-oxazolineco-2-butyl-2-oxazoline) block and above CPT they form polymeric micelles with diameter $\sim 200 \mathrm{~nm}$. The phenolic moiety introduced into the copolymer allowed radionuclide labeling with ${ }^{125} \mathrm{I}$ with sufficient in vitro stability under model conditions. Various iodine radioisotopes are suitable for both diagnosis and radionuclide therapy of solid tumors. The composition of these triblock copolymers is more complex than that of the vast majority of poly(2-alkyl-2-oxazoline)s: a statistical thermoresponsive (iPrOx) and hydrophobic (BuOx) central block with terminal hydrophilic blocks (MeOx). As temperature increases, nanoparticles are formed in a process starting with single molecules that become loose aggregates and ends with the formation of compact nanoparticles. It has been proven (Bogomolova et al. 2014) that the (iPrOx $+\mathrm{BuOX}) / \mathrm{MeOx}$ ratio determines the value of the cloud point temperature, whereas the different $\mathrm{BuOx}-\mathrm{iPrOx}$ blocks determines the character of the process.

The $\mathrm{pH}$-sensitive polymers have attracted a considerable research interest because the differences in $\mathrm{pH}$ between normal tissue and cancer tissues create an opportunity to design $\mathrm{pH}$-sensitive drug delivery systems that can target tumors and release loaded drugs at the tumor site (Liua et al. 2013, Zhang et al. 2014). Great effort was devoted to biodegradable polymeric micelles because of their degradability prevents micelle accumulation that could results in long term toxicity. The employment of $\mathrm{pH}$-responsive micelles is another approach because the polymers may be triggered and destabilized in response to the slightly acidic tumor 
microenvironment, enabling rapid release of active agents upon arrival at the desired site and simplifying renal clearance. In such way, we recently devised block copolymers to produce nanoparticles comprising a PDPA (poly[2-(diisopropylamino)ethyl methacrylate]) core. PDPA is a promising smart material for the construction of tumor targeting drug delivery polymeric nanocarriers because it is able to encapsulate hydrophobic anticancer drugs and it undergoes a sharp hydrophobic-hydrophilic $\mathrm{pH}$-induced transition within a $\mathrm{pH}$ range that is desirable for tumor-targeting drug delivery (Giacomelli et al. 2011). PDPA is one of the few polymers that can quickly release hydrophobic guest molecules at specific tumor sites via a pH-triggered pathway at slightly acidic conditions also degrading micelles into unimers with molecular weight below renal threshold and thus eliminable by kidneys (Pegoraro et al. 2013, Wang et al. 2014).

The block copolymer poly[ethylene oxide-bglycerol monomethacrylate-b-2-(diisopropylamino)ethyl methacrylate] self-assembles in phosphate buffered saline (PBS) into highly regular spherical micelles. The micellar size (diameter $42 \mathrm{~nm}$ ) and micellar molecular weight $\left(\mathrm{M}_{\mathrm{w}}>100 \mathrm{kDa}\right)$ are in the range to avoid renal clearance providing a long blood circulation time. Their size is below the cut-off size of the leaky pathological vasculature $(\mathrm{d}<200 \mathrm{~nm})$, making those candidates for the use in cancer therapy based on the EPR (Enhanced Permeation and Retention) effect (Maeda et al. 2000). The $\mathrm{pH}$-responsive PDPA core could be loaded with the poorly water-soluble anticancer drug paclitaxel (PTX) with encapsulation efficiency $\sim 70 \%$ and drug loading content $\sim 7 \%$.

Some attention was paid to complexation of block copolymers of (hydrogen donor)-block-(hydrogen acceptor) by intermolecular hydrogen bonding (Oyama et al. 1989, Hemker et al. 1990, Poe et al. 2004, Burova et al. 2005). Recently, formation of interpolyelectrolyte complexes by mixing poly(2-vinylpyridine-blockethylene oxide) with neutralized poly(methacrylic acidblock-ethylene oxide) (PMAA-b-PEO) in water was investigated (Gohy et al. 2001). They observed as a side effect that PMAA-b-PEO copolymers consisting of long PEO blocks and short PMAA blocks aggregate at low $\mathrm{pH}$ into micelles with a narrow size distribution and typical hydrodynamic radius $\mathrm{R}_{\mathrm{h}}=55 \mathrm{~nm}$, the core of which is formed by a hydrogen-bonded complex between PMAA and PEO blocks and the shell is formed by uncomplexed PEO chain sections. These micelles form only in a temperature range $15-50{ }^{\circ} \mathrm{C}$ with maximal stability at
$40{ }^{\circ} \mathrm{C}$. PMAA-b-PEO copolymers with long PMAA blocks and short PEO blocks were investigated in (Holappa et al. 2004, Konak and Sedlak 2007, Seanger 1984). If the MAA block is sufficiently long compared to the PEO block, stable micelles with narrow size distribution were formed in water at low $\mathrm{pH}$. The complexes are stabilized by excess of uncomplexed MAA units.

We have investigated (Konak and Sedlak 2007) the $\mathrm{pH}$-sensitive micelles formed by interchain hydrogen bonding of PMAA-b-PEO copolymers at $\mathrm{pH}<5$. Both the molecular weight $M_{w}$ and the hydrodynamic radius $R_{h}$ of micelles increase with decreasing $\mathrm{pH}$ of solution, displaying an asymptotical tendency at low $\mathrm{pH}$ values. The observed micelles are well defined nanoparticles with narrow size distributions (polydispersity $\Delta R_{h} / R_{h} \leq 0.05$ ) comparable to regular diblock copolymer micelles. The proposed structure of the micelles is schematically represented in Figure 3. The critical micellar concentration (cmc) occurs slightly below $\mathrm{c}=1 \times 10^{-4} \mathrm{~g} \mathrm{ml}^{-1}$. The micelles are slightly negatively charged.

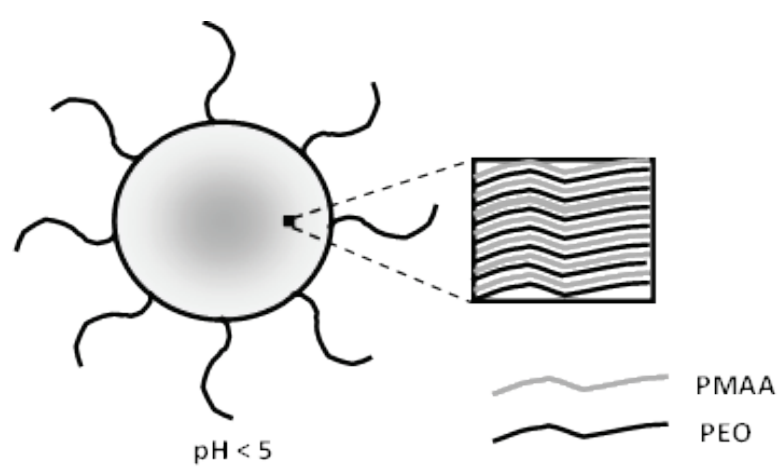

Fig. 3. Presumed structure of hydrogen-bonded nanoparticles.

The combination of hybrid hydrogen bonds and cooperative phenomena is also characteristic of polynucleotides, where the complementary bases form a specific pair through the stereoselective hydrogen bonds. The hybrid hydrogen bonds govern the DNA double helix formation (Saenger 1984). A similar mechanism was used for formation of macromolecular complexes of single stranded RNA and DNA with polysaccharides (Sakuray et al. 2001) and proteins (Jayaram et al. 2001).

A new biocompatible and biodegradable diblock copolymer (Petrova et al. 2014) that contains a specific acid-labile degradable linkage (acyclic ketal group) 
between the hydrophobic poly( $\varepsilon$-caprolactone) (PCL) and the hydrophilic poly(ethylene oxide monomethyl ether) (MPEO) block was prepared. Upon dissolution in a mild organic solvent, the MPEO-b-PCL block copolymer selfassembled in water-PBS into regular, spherical, stable nanoparticles. Furthermore, the presence of the acidlabile ketal linker enabled the disassembly of these nanoparticles in a buffer that simulated acidic cytosolic or endosomal conditions in tumour cells. This disassembly led to hydrolysis profiles that resulted in neutral degradation products.

The diblock copolymer monomethoxy-PEO-bpoly(D,L-lactide) (MPEO-PDLLA) is used for approved micellar formulation of anticancer drug paclitaxel for the treatment of cancer in clinical studies (Cabral and Kataoka 2014). In a similar way, paclitaxel-loaded micellar systems prepared from PEO-poly(aspartic acid) (PEO-b-Pas) are used in clinical trial of gastric cancer (Kato et al. 2012). Diblock copolymers made of 6-Omethacryloyl-1,2:3,4-di-O-isopropylidene-D-galactopyranose (MAIGal) and 2-(dimethylamino)ethyl methacrylate (DMAEMA) were used to construct spherical brushes with a crosslinked polystyrene core (Arslan et al. 2014). Variation of $\mathrm{pH}$ makes possible to reversibly expose to the external medium the inner block attached to the nanoparticle surface. This property makes these nanoparticles to be a very useful model of a delivery system specifically targeted to the liver for treatment of hepatocellular carcinoma. In the bloodstream at $\mathrm{pH} \sim 7.4$ the PDMAEMA block is collapsed, the glycopolymer block is exposed to the outside and is selectively accumulated in liver due to uptake via asialoglycoprotein receptor. After contact with the tumor that has $\mathrm{pH} \sim 5$, the PDMAEMA block will expand and its newly exposed cationic surface will damage the anionic surface of the cancer cells.

\section{Nanoparticles from statistical copolymers}

Graft and statistical copolymers can also be considered as special cases of block copolymers. In the first case, B blocks are attached to the A backbone either regularly or randomly. In the second case, the polymer is a linear chain, the alternating A and B blocks are short and have a random length. These polymers in many cases do not form micelles or other self-assembled systems spontaneously but can be used to create nanoparticles applicable for biomedical purposes by various physicochemical procedures such as emulsification
(Weikun et al. 2011), complexation (Mueller 2014) or nanoprecipitation (Schubert et al. 2011). The latter technique has become quite popular in the past years since it allows controlled preparation of well-defined nanoparticles.

Polymer nanoparticles prepared by selfassembling thermoresponsive poly[ $\mathrm{N}$-isopropylacrylamide)-graft-N-(2-hydroxypropyl)methacrylamide] copolymers with hydrolytically degradable N-glycosylamine groups between the polymer blocks were prepared (Hruby et al. 2009) for delivery of diagnostic and therapeutic radionuclides into solid tumors. The nanoparticles are formed by fast heating of an aqueous solution of the copolymer to $37.8^{\circ} \mathrm{C}$. They have a hydrodynamic diameter of $128 \mathrm{~nm}$ measured by dynamic light scattering and slowly degrade during incubation in aqueous buffer at $\mathrm{pH}=7.4$. Labeling with both ${ }^{131} \mathrm{I}$ and ${ }^{90} \mathrm{Y}$ proceeds with high yields $(>85 \%)$. The unlabeled polymers are not cytotoxic for any of the tested murine and human cell lines.

Statistical copolymers of poly[N-(2hydroxypropyl)methacrylamide] with a different amount of cholesterol moieties attached to the backbone by a non-cleavable spacer have been studied previously (Filippov et al. 2012, Filippov et al. 2013). Additionally, some of the copolymers were modified with anticancer drug doxorubicin (Dox) bound via the $\mathrm{pH}$-sensitive hydrazone bond. Dilute solutions of conjugates in phosphate buffer at $\mathrm{pH}=5.0$ and 7.2 were examined by dynamic and static light scattering (DLS/SLS), smallangle X-ray and neutron scattering - SAXS and SANS. It was proven that the presence of any amount of cholesterol results in formation of anisotropic nanoparticles. The obtained results show that the size and anisotropy of the nanoparticles grow with increasing amount of cholesterol moieties.

The SAXS and SANS experiments allowed determination of the three-dimensional structure of nanoparticles composed of cholesterol conjugates of poly[N-(2-hydroxypropyl)methacrylamide]. From $a b$ initio calculations and the shape of pair-distance distribution function together with the shape of crosssectional pair-distance distribution function it was concluded that the most probable structure of nanoparticles is the pearl-necklace one where ellipsoidal pearls composed of cholesterol mainly are connected by bridges composed of hydrophilic HPMA copolymer chains.

Thermoresponsive statistical polyoxazoli- 
nes, poly[(2-butyl-2-oxazoline)-stat-(2-isopropyl-2-oxazoline)] [pBuOx-co-piPrOx] with different hydrophobic moieties were combined with the FDA-approved triblock copolymer Pluronic F127 (PEO-PPO-PEO) as a template system for the creation of thermosensitive nanoparticles (Bogomolova et al. 2013). It was shown that the presence of the thermosensitive F127 triblock copolymer in solution reduces nanoparticle size and polydispersity. When the temperature is raised above the CPT, hybrid nanoparticles composed of both the polyoxazolines and F127 are predominant in solution. The molecular weight and hydrophobicity of the polymer do not influence the size of the outer radius and only slightly change the inner radius of the nanoparticles. Poly(2-oxazoline) molecules are fully incorporated inside of F127 micelles, and this result is very promising for the successful application of such systems in delivery of radionuclides and bioactive compounds. Pluronic F127 and some similar polyamphiphiles are also known to be inhibitors of $\mathrm{ABC}$ cassette transporters such as P-glycoprotein, overexpression of which causes multidrug resistance of tumor cells. Therefore, such copolymers are able to restore sensitivity of the cancer cells towards chemotherapy.

An interesting group of thermoresponsive graft copolymers was prepared (Pospisilova et al. 2014) and is based on glycogen-graft-poly(2-alkyl-2-oxazolines). These nanostructured hybrid dendrimeric stimuliresponsive polymers combine the body's own biodegradable polysaccharidic dendrimer glycogen with the widely tunable thermoresponsive poly(2-alkyl-2oxazoline)s, the latter being polypeptide analogs and are known to be biocompatible. Glycogen-graft-poly(2-alkyl2-oxazoline)s were prepared by a simple one-pot two-step procedure involving cationic ring-opening polymerization of 2-alkyl-2-oxazolines followed by termination of the living cationic ends with sodium glycogenate. As confirmed by light and X-ray scattering, as well as cryo transmission electron microscopy, the grafted dendrimer structure allows easy adjustment of the cloud point temperature and the nanostructure of the self-assembled phase separated polymer by the graft composition, length and the grafting density.

Aliphatic biodegradable copolyesters are a very promising group of copolymers very promising for biologically applicable nanoparticles. Surfactant-free, narrowly distributed, nanosized spherical particles $\left(\mathrm{R}_{\mathrm{h}}<60 \mathrm{~nm}\right)$ have been produced from the poly(butylene succinate-co-butylene dilinoleate) (PBS/PBDL) by a single-step nanoprecipitation method (Jager et al. 2012). Combined SLS and DLS measurements suggested that the nanoparticles comprise a porous core. The nanoparticles loaded with 6-7\% paclitaxel had a pronounced stability and relatively rapid degradation. Cell viability experiments demonstrated that the nanoparticles are fully biocompatible and non-toxic, making them useful for biomedical applications.

Their porosity of nanoparticles enables water to be entrapped which is responsible for their pronounced stability and relatively fast degradation as followed by size exclusion chromatography (SEC). The polymeric nanoparticles could be loaded with the hydrophobic model drug paclitaxel (PTX) with an encapsulation efficiency of $\sim 95 \%$ and drug loading content of 6-7 \%. The drug encapsulation and release modifies the inner structure of the nanoparticles, which holds a large amount of entrapped water in the drug-free condition. PTX encapsulation leads to replacement of the entrapped water by the hydrophobic model drug and to shrinking of the nanoparticles, due to favorable drug-polymer hydrophobic interactions. Cell viability experiments demonstrated that the nanoparticles are biocompatible and non-toxic, making them potentially useful for applications in nanomedicine.

\section{Conclusions}

Block copolymers forming supramolecular assemblies in aqueous media represent materials which are extremely useful for the construction of drug delivery systems especially for cancer applications. The nanoparticle formulations suppress unwanted physicochemical properties of the encapsulated drugs (such as poor water solubility, unfavorable distribution, e.g. entrapment in reticuloendotelial system, or too fast renal elimination), modify biodistribution of the drugs towards targeted delivery into tissue of interest and allow triggered release of the active cargo.

\section{Conflict of Interest}

There is no conflict of interest.

\section{Acknowledgements}

The work was supported by the Ministry of Education, Youth and Sports of CR within the National Sustainability Program I (NPU I), Project POLYMAT LO1507. 


\section{References}

ARSLAN H, PFAFF A, LU Y, STEPANEK P, MÜLLER AH: Stimuli-responsive spherical brushes based on D-galactopyranose and 2-(dimethylamino)ethyl methacrylate. Macromol Biosci 14: 81-91, 2014.

ASEYEV V, TENHU H, WINNIK FM: Temperature-dependence of the colloidal stability of neutral amphiphilic polymers in water. Adv Polym Sci 196, 1-85, 2006.

BOGOMOLOVA A, FILIPPOV SK, STAROVOYTOVA L, ANGELOV B, KONAREV P, SEDLACEK O, HRUBY M, STEPANEK P: Study of complex thermosensitive amphiphilic Polyoxazolines and their interaction with ionic surfactants. Are hydrophobic, thermosensitive, and hydrophilic moieties equally important? J Phys Chem B 118: 4940-4950, 2014.

BOGOMOLOVA A, HRUBY M, PANEK J, RABYK M, TURNER S, BALS S, STEINHART M, ZHIGUNOV A, SEDLACEK O, STEPANEK P, FILIPPOV SK: Small-angle X-ray scattering and light scattering study of hybrid nanoparticles composed of thermoresponsive triblock copolymer F127 and thermoresponsive statistical polyoxazolines with hydrophobic moieties. J Appl Crystallogr 46: 1690-1698, 2013.

BOGOMOLOVA A, KELLER S, KLINGLER J, SEDLAK MC, RAK D, STURCOVA A, HRUBY M, STEPANEK P, FILIPPOV SK: Self-assembly thermodynamics of pH-responsive amino-acid-based polymers with a nonionic surfactant. Langmuir 30: 11307-11318, 2014.

BUROVA TV, GRINBERG NV, GRINBERG VY, KALININA VE, LOZINSKY VI, ASEYEV VO, HOLAPPA S, TENHU H, KHOKHLOV AR: Unusual conformational behavior of complexes of poly $(\mathrm{N}-$ isopropylacrylamide) with poly(methacrylic acid). Macromolecules 38: 1292-1299, 2005.

CABRAL H, KATAOKA K. Progress of drug-loaded polymeric micelles into clinical studies. $J$ Control Release 190: 465-476, 2014.

CAHN JW: The kinetics of grain boundary nucleated reactions. Acta Metall 4: 449-459, 1956.

CAMMAS S, SUZUKI K, SONE C, SAKURAI Y, KATAOKA K, OKANO T: Thermo-responsive polymer nanoparticles with a core-shell micelle structure as site-specific drug carriers. $J$ Contr Release 48: 157-164, 1997.

CHRISTIAN JW: The Theory of Transformations in Metals and Alloys. Part I, Pergamon, New York 1981.

CHYTRY V, NETOPILIK M, BOHDANECKY M, ULBRICH K: Phase transition parameters of potential thermosensitive drug release systems based on polymers of N-alkymethacrylamides. J Biomater Sci Polym Ed 8: 817-824, 1997.

FILIPPOV SK, FRANKLIN JM, KONAREV PV, CHYTIL P, ETRYCH T, BOGOMOLOVA A. DYAKONOVA M, PAPADAKIS CM, RADULESCU A, ULBRICH K, STEPANEK P, SVERGUN DI: Hydrolytically degradable polymer micelles for drug delivery: A SAXS/SANS kinetic study. Biomacromolecules 14: 40614070, 2013.

FILIPPOV S, HRUBY M, KONAK C, MACKOVA H, SPIRKOVA M, STEPANEK P: Novel pH-responsive nanoparticles. Langmuir 24: 9295-9301, 2008.

FILIPPOV SK, CHYTIL P, DYAKONOVA M, PAPADAKIS CM, JIGOUNOV A, PLESTIL J, STEPANEK P, ETRYCH T, ULBRICH K, SVERGUN DI: Macromolecular HPMA-based nanoparticles with cholesterol for solid-tumor targeting: detailed study of the inner structure of a highly efficient drug delivery system. Biomacromolecules 13: 2594-2604, 2012.

FORSTER S, KONRAD M: From self-organizing polymers to nano- and biomaterials. J Mater Chem 13: 2671-2688, 2003.

GIACOMELLI FC, STEPANEK P, GIACOMELLI C, SCHMIDT V, JÄGER E, JÄGER A, ULBRICH K: pHtriggered block copolymer micelles based on a pH-responsive PDPA (poly[2-(diisopropylamino)ethyl methacrylate])inner Core and a PEO (poly(ethylene oxide)) outer shell as a potential tool for the cancer therapy. Soft Matter 7: 9316-9325, 2011.

GOHY JF, VARSHNEY SK, JEROME R: Water-soluble complexes formed by poly(2-vinylpyridinium)-blockpoly(ethylene oxide) and poly(sodium methacrylate)-block-poly(ethylene oxide) copolymers. Macromolecules 34: 3361-3366, 2001. 
HADJICHRISTIDIS N, PISPAS S, FLOUDAS G: Block Copolymers: Synthetic Strategies, Physical Properties, and Applications. Wiley-Interscience, 2002.

HAMLEY IW: Physics of Block Copolymers, Oxford University Press, 1999.

HEMKER DJ, GARZA V, OYAMA HT, FRANK CW: Complexation of poly(acrylic acid) and poly(methacrylic acid) with pyrene end-labeled poly(ethylene glycol): $\mathrm{pH}$ and fluorescence measurements. Macromolecules 23: 44114418, 1990.

HIROTSU S, HIROKAWA Y, TANAKA T: Volume-phase transitions of ionized N-isopropylacrylamide gels. $J$ Chem Phys 87: 1392-1395, 1987.

HOFFMAN AS: Inteligent polymers. In: Controlled Drug Delivery. PARK K (ed.), ACS Publications, Washington, 1997, pp 485-498.

HOLAPPA S, KANTONEN L, WINNIK FM, TENHU H: Self-complexation of poly(ethylene oxide)-blockpoly(methacrylic acid) studied by fluorescence spectroscopy. Macromolecules 37: 7008-7018, 2004.

HRUBY M, FILIPPOV SK, PANEK J, NOVAKOVA M, MACKOVA H, KUCKA J, ULBRICH K: Thermoresponsive micelles for radionuclide delivery. $J$ Control Release 148: e60-e62, 2010a.

HRUBY M, FILIPPOV SK, PANEK J, NOVAKOVA M, MACKOVA H, KUCKA J, VETVICKA D, ULBRICH K: Polyoxazoline thermoresponsive micelles as radionuclide delivery systems. Macromol Biosci 10: 916-924, 2010b.

HRUBY M, KONAK C, KUCKA J, VETRIK M, FILIPPOV SK, VETVICKA D, MACKOVA H, KARLSSON G, EDWARDS K, RIHOVA B, ULBRICH K: Thermoresponsive, hydrolytically degradable polymer micelles intended for radionuclide delivery. Macromol Biosci 9: 1016-1027, 2009.

HRUBY M, KONAK C, ULBRICH K: Polymeric micellar pH-sensitive drug delivery system for doxorubicin. J Control Release 103: 137-148, 2005a.

HRUBY M, KONAK C, ULBRICH K: Poly(allyl glycidyl ether)-block-poly(ethylene oxide): A Novel Promising polymeric intermediate for the preparation of micellar drug delivery systems. J Appl Polym Sci 95: 201-211, $2005 b$.

HRUBY M, KONAK C, ULBRICH K: Poly(ethylene oxide)-coated polyamide nanoparticles degradable by glutathione. Colloid Polym Sci 285: 569-574, 2007.

JAIN KK: Drug Delivery in Cancer: Technologies and Commercial Opportunities. Decision Resources, Inc., Waltham, U.S.A., 2000.

JAYARAM B, McCONNELL, DIXIT SB, BEVERIDGE DL: Free-energy component analysis of 40 protein-DNA complexes: a consensus view on the thermodynamics of binding at the molecular level. J Comput Chem 23: $1-14,2001$.

JÄGER A, GROMADZKI D, JÄGER E, GIACOMELLI FC, KOZLOWSKA A, KOBERA L, BRUS J, RIHOVA B, EL FRAY M, ULBRICH K, STEPANEK P: Novel "soft" biodegradable nanoparticles prepared from aliphatic based monomers as a potential drug delivery system. Soft Matter 8: 4343-4354, 2012.

JEONG B, KIM SW, BAE YH: Thermosensitive sol-gel reversible hydrogels. Adv Drug Deliv Rev 54: 37-51, 2002.

SEYMOUR LW, KATAOKA K, KABANOV AV: Cationic block copolymers as self-assembling vectors for gene delivery. In: Self-assembling Complexes for Gene Delivery: From Laboratory to Clinical Trials. KABANOV KV, FELGNER PL, SEYMOUR LW (eds), John Wiley \& Sons, New York, 1998, pp 219-237.

KATAOKA K, HARADA A, NAGASAKI Y: Block copolymer micelles for drug delivery: Design, characterization and biological significance. Adv Drug Del Rev 47: 113-131, 2001.

KATAOKA K, MATSUMOTO T, YOKOYAMA M, OKANO T, SAKURAI Y, FUKUSHIMA S, OKAMOTO K, KWON GS: Doxorubicin-loaded poly(ethylene glycol)-poly(beta-benzyl-L-aspartate) copolymer micelles: their pharmaceutical characteristics and biological significance. $J$ Control Release 64: 143-153, 2000.

KATO K, CHIN K, YOSHIKAWA T, YAMAGUCHI K, TSUJI Y, ESAKI T, SAKAI K, KIMURA M, HAMAGUCHI T, SHIMADA Y, MATSUMARA Y, IKEDA R: Phase II Study of NK105, a Paclitaxel incorporating micellar nanoparticle, for previously treated advanced or recurrent gastric cancer. Invest New Drugs 30: 1621-1627, 2012.

KNOP K, HOOGENBOOM R, FISCHER D, SCHUBERT US: Poly(ethylene glycol) in drug delivery: Pros and cons as well as potential alternatives. Angew Chem-Int Edit 49: 6288-6308, 2010. 
KOHORI F, SAKAI K, AOYAGI T, YOKOYAMA M, SAKURAI Y, OKANO T: Preparation and characterization of thermally responsive block copolymer micelles comprising poly(N-isopropylacrylamide-b-DL-lactide). J Control Release 55: 87-98, 1998.

KONAK C, KOPECKOVA P, KOPECEK J: Photoregulated adsorption and association of amphiphilic copolymers containing azobenzene side chains. J Colloid Interface Sci 168: 235-241, 1994.

KONAK C, RATHI RS, KOPECKOVA P, KOPECEK J: Photoregulated association of water-soluble copolymers with spirobenzopyran-containing side chains. Macromolecules 30: 5553-5556, 1997.

KONAK C, HRUBY M: Thermoresponsive Polymeric Nanoemulsions. Macromol Rapid Commun 27: 877-881, 2006.

KONAK C, PANEK J, HRUBY M: Thermoresponsive polymeric nanoparticles stabilized by surfactants. Colloid Polym Sci 285: 1433-1439, 2007.

KONAK C, SEDLAK M: pH-Sensitive micelles formed by interchain hydrogen bonding of poly(methacrylic acid)block-poly(ethylene oxide) copolymers. Macromol Chem Phys 208: 1893-1899, 2007.

KUJAWA P, ASEYEV V, WINNIK FM, TENHU H: Temperature-sensitive properties of poly(n-isopropylacrylamide) mesoglobules formed in dilute aqueous solutions heated above their demixing point. Macromolecules 39: 7686-7693, 2006.

KWON GS, KATAOKA K: Block copolymer micelles as long-circulating drug vehicles. Adv Drug Deliver Rev 16: 295-309, 1995.

KWON GS, OKANO T: Polymeric micelles as new drug carriers. Adv Drug Deliver Rev 21: 107-116, 1996.

LAZZARI M, LIU G, LACOMMANDOU S: Block Copolymers in Nanoscience. John Wiley \& Sons, New York, 2006.

LEE ES, NA K, BAE YH: Super pH-sensitive multifunctional polymeric micelle. Nano Lett. 5: 325-329, 2005.

LEE HI, PIETRASIK J, MATYJASZEWSKI K: Phototunable temperature-responsive molecular brushes prepared by ATRP. Macromolecules 39: 3914-3920, 2006.

LEROUX J-Ch, ROUX E, LE GARREC D, HONG K, DRUMMOND DC: N-isopropylacrylamide copolymers for the preparation of pH-sensitive liposomes and polymeric micelles. J Control Release 72: 71-84, 2001.

LIANG D, ZHOU S, SONG L, ZAITSEV VS, CHU B: Copolymers of poly(n-isopropylacrylamide) densely grafted with poly(ethylene oxide) as high-performance separation matrix of DNA. Macromolecules 32: 6326-6332, 1999.

LICCIARDI M, GIAMMONA G, DU J, ARMES SP, TANG Y, LEWIS AL: New folate-functionalised biocompatible block copolymer micelles as potential anti-cancer drug delivery systems. Polymer 47: 2946-2955, 2006.

LIUA Y, WANGA W, YANGA J, ZHOUA CH, SUN J: pH-sensitive Polymeric Micelles Triggered Drug Release for Extracellular and Intracellular Drug Targeting Delivery. Asian J Pharm Sci 8: 159-167, 2013.

MAEDA H, WU J, SAWA T, MATSUMURA Y, HORI K: Tumor vascular permeability and the EPR effect in macromolecular therapeutics: a review. J Control Release 65: 271-284, 2000.

MONJI N, HOFFMAN AS: A novel immunoassay system and bioseparation process based on thermal phase separating polymers. Appl Biochem Biotechnol 14: 107-120, 1987.

MUELLER M: Polyelectrolyte Complexes in the Dispersed and Solid State. Springer Verlag, Heidelberg, 2014.

OKANO T, YAMADA N, OKUHARA M, SAKAI H, SAKURAI Y: Mechanism of cell detachment from temperaturemodulated, hydrophilic-hydrophobic polymer surfaces. Biomaterials 16: 297-303, 1995.

OUPICKY D, RESCHEL T, KONÁK C, OUPICKA L: Temperature controlled behavior of self-assembly gene delivery vectors based on complexes of DNA with poly(L-lysine)-graft-poly(N-isopropylacryl amide). Macromolecules 36: 6863-6872, 2003.

OYAMA HT, HEMKER DJ, FRANK CW: Effect of the degree of ionization of poly(methacrylic acid) on the complex formed with pyrene end-labeled poly(ethylene glycol). Macromolecules 22: 1255, 1989.

PARK TG, HOFFMAN AS: Effect of temperature cycling on the activity and productivity of immobilized betagalactosidase in a thermally reversible hydrogel bead reactor. Appl Biochem Biotechnol 19: 1-9, 1988.

PEGORARO C, CECCHIN D, GRACIA LS, WARREN N, MADSEN J, ARMES SP, LEWIS A, MACNEIL S, BATTAGLIA G: Enhanced drug delivery to melanoma cells using PMPC-PDPA polymersomes. Cancer Lett 334: 328-337, 2013. 
PETROVA S, JAGER E, KONEFAL R, JAGER A, DE GARCIA VENTURINI C, SPEVACEK J, STEPANEK P: Novel poly(ethylene oxide monomethyl ether)-b-poly(.epsilon.-caprolactone) diblock copolymers containing a pH-acid labile ketal group as a block linkage. Polym Chem 5: 3884-3893, 2014.

POE GD, JARRETT WL, SCALES CW, MCCORMICK CL: enhanced coil expansion and intrapolymer complex formation of linear poly(methacrylic acid) containing poly(ethylene glycol) grafts. Macromolecules 37: 26032612, 2004.

POSPISILOVA A, FILIPPOV SK, BOGOMOLOVA A, TURNER S, SEDLACEK O, MATUSHKIN N, CERNOCHOVA Z, STEPANEK P, HRUBY M: Glycogen-graft-poly(2-alkyl-2-oxazolines) - the new versatile biopolymer-based thermoresponsive macromolecular toolbox. RSC Adv 4: 61580-61588, 2014.

SAIYTO S, KONNO M, INOMATA H: Volume phase transition of N-alkylacrylamide gels. Adv Polym Sci 109: $207-$ 232, 1993.

SAKAI K, SMITH EG, WEBBER GB, SCHATZ C, WANLESS EJ, BÜTÜN V, ARMES SP, BIGGS S: $\mathrm{pH}$-responsive diblock copolymer micelles at the silica/aqueous solution interface: Adsorption kinetics and equilibrium studies. J Phys Chem B 110: 14744-14753, 2006.

SAKURAY K, MIZU M, SHINKAI S: Polysaccharide--polynucleotide complexes. 2. Complementary polynucleotide mimic behavior of the natural polysaccharide schizophyllan in the macromolecular complex with singlestranded RNA and DNA. Biomacromolecules 2: 641-650, 2001.

SCHILD HG: Poly(N-isopropylacrylamide): Experiment, theory and application. Prog Polym Sci 17: 163-249, 1992.

SCHUBERT S, DELANEY JT Jr, SCHUBERT US: Nanoprecipitation and nanoformulation of polymers: from history to powerful possibilities beyond poly(lactic acid). Soft Matter 7: 1581-1588, 2011.

SAENGER W: Forces stabilizing associations between bases: Hydrogen bonding and base stacking. In: Principles of Nucleic Acid Structure. Springer-Verlag, New York, 1984, pp 116-158.

SEDLACEK O, MONNERY BD, FILIPPOV SK, HOOGENBOOM R, HRUBY M: Poly(2-Oxazoline)s - are they more advantageous for biomedical applications than other polymers? Macromol Rapid Commun 33: 1648$1662,2012$.

SHIMOBOJI T, LARENAS E, FOWLER T, KULKARNI S, HOFFMAN S: Photoresponsive polymer-enzyme switches. Proc Natl Acad Sci USA 99: 16592-16596, 2002.

STROBL G. The Physics of Polymers: Concepts for Understanding Their Structures and Behavior. Springer-Verlag Berlin Heidelberg, 2007.

SWARBICH J: Colloidal Drug Delivery Systems in Drug and Pharmaceutical Sciences. Marcel Dekker, New York, 1994.

TAYLOR LD, CERENKOWSKI LD: Preparation of films exhibiting a balanced temperature dependence to permeation by aqueous solution. Lower consolute behavior. J Polym Sci 13: 2551-2570, 1975.

TORCHILIN VP: PEG-based Micelles as carriers of contrast agents for different imaging modalities. Adv Drug Deliv Rev 54: 235-252, 2002.

WANG G, TONG X, ZHAO Y: Preparation of azobenzene-containing amphiphilic diblock copolymers for lightresponsive micellar aggregates. Macromolecules 37: 8911-8917, 2004.

WANG X, WU C: Light-scattering study of coil-to-globule transition of a poly(n-isopropylacrylamide) chain in deuterated water. Macromolecules 32: 4299-4301, 1999.

WANG Y, ZHOU K, HUANG G, HENSLEY C, HUANG X, MA X, ZHAO T, SUMER BD, DEBERARDINIS RJ, GAO JA: Nanoparticle based strategy for the imaging of a broad range of tumours by nonlinear amplification of microenvironment signals. Nat Mater 13: 204-212, 2014.

WEIKUN L, SHANQIN L, RENHUA D, JINTAO Z: Encapsulation of nanoparticles in block copolymer micellar aggregates by directed supramolecular assembly. Angew Chem-Int Edit 50: 5865-5868, 2011.

YOO HS, PARK TG: Biodegradable polymeric micelles composed of doxorubicin conjugated PLGA-PEG block copolymer. J Control Rel 70: 63-70, 2001.

YOO HS, LEE EA, PARK TG: Doxorubicin-conjugated biodegradable polymeric micelles having acid-cleavable linkages. J Control Rel 82: 17-27, 2002.

YU J, TAO X, TAM H, YANG D, DEMOKAN M: Photosensitivity and grating development in trans-4stilbenemethanol-doped poly(methyl methacrylate) materials. Optics Commun 265: 132-139, 2006. 
ZHANG Z, SUN Q, ZHONG J, YANG Q, LI H, DU C, LIANG B, SHUAI X: Magnetic resonance imaging-visible and pH-sensitive polymeric micelles for tumor targeted drug delivery. J Biomed Nanotechnol 10: 216-226, 2014. 